Revista de Ensino em Artes, Moda e Design

Dossiê 3

Novas perspectivas de aprendizagem

\title{
O TRABALHO DOCENTE UNIVERSITÁRIO COMO PRÁTICA RELACIONAL: ASSUNTOS, SABERES E INSTITUIÇÕES
}

\section{El trabajo docente universitario como práctica relacional: sujetos, saberes e instituciones ${ }^{1}$}

\section{Verónica Soledad Walker ${ }^{2}$}

\footnotetext{
1 Este artigo foi originalmente publicado pela revista Revista Educación, el Lenguaje y la Sociedad (FCH, UNLPam). Disponível em: <https://cerac.unlpam.edu.ar/index.php/els/article/view/2219/2216>.

2 Doutora pela Universidade de Malaga. Atua na Universidade Nacional do Sul, Argentina. Suas pesquisas têm como ênfase Educação e sociedade.

E-mail: veroswalker@gmail.com
} 


\section{Resumo}

Este artigo discute a dimensão relacional do trabalho docente universitário a partir da análise da bibliografia especializada e das informações coletadas por meio de entrevistas realizadas por professores universitários argentinos e espanhóis. Sugere-se que o trabalho docente constitua uma prática social que coloca os professores em um quadro de múltiplas relações com outros sujeitos, conhecimentos específicos, instituições particulares e com a sociedade em geral. Ao longo da escrita, analisam-se as relações entre colegas e as formas adquiridas pela cultura do trabalho docente na Universidade; As características que a ligação estudantil assume; $O$ vínculo entre os professores e a instituição universitária e a organização da sociedade, bem como a relação com certos saberes que requerem o exercício do trabalho docente universitário. O reconhecimento da rede de múltiplos relacionamentos, dos quais os professores exercem seu trabalho cotidiano, permite que pensem no ensino como uma prática heterogênea atravessada por múltiplas tensões.

Palavras-Chave: Universidade, trabalho docente, instituições, saberes, relações.

\section{Resumen}

El presente artículo aborda la dimensión relacional del trabajo docente universitario a partir del análisis de la bibliografía especializada y de la información recogida a través de entrevistas realizadas a profesores universitarios argentinos y españoles. Se plantea que el trabajo docente constituye una práctica social que coloca a los profesores en una trama de múltiples relaciones con otros sujetos, saberes específicos, instituciones particulares y con la sociedad en general. A lo largo del escrito, se analizan las relaciones entre colegas y las formas que adquiere la cultura del trabajo docente en la universidad; las características que asume el vínculo con estudiantes; el vínculo de los docentes con la institución universitaria y la organización de pertenencia, así como la relación con determinados saberes que exige el ejercicio del trabajo docente universitario. El reconocimiento de la red de múltiples relaciones desde la cual los/as docentes ejercen su quehacer cotidiano, habilita a pensar el trabajo docente como una práctica heterogénea atravesada por múltiples tensiones.

Palabras clave: Universidad, Trabajo docente, Instituciones, Saberes, Relaciones. 


\section{INTRODUCCIÓN}

El trabajo docente universitario constituye una práctica compleja, heterogénea y específica que es vivida y percibida diferencialmente por los docentes en función de sus particulares posiciones en el campo universitario. En el marco de condiciones materiales y simbólicas diversas, el desarrollo del trabajo docente en la universidad coloca a los profesores en una red de múltiples relaciones. En primer lugar, desempeñarse como docente universitario implica relacionarse con una organización particular que contrata para el ejercicio de una función institucional. En segundo lugar, supone establecer relaciones con colegas que - posicionados diferencialmente y no necesariamente con la misma relación de contratación- están convocados para el cumplimiento de determinadas funciones institucionales. En tercer lugar, el trabajo docente conlleva relacionarse prioritariamente con aquellos que son los destinatarios directos de la tarea de enseñanza: Ios estudiantes. En cuarto lugar, desempeñarse como docente universitario comprende entablar relaciones con saberes de tipo disciplinar, pedagógico y experiencial que se ponen en juego en el ejercicio del quehacer cotidiano. De modo que el trabajo docente se define como práctica relacional en tanto supone el despliegue de múltiples relaciones sobre la base de los vínculos que la propia institución universitaria establece con la sociedad.

El presente artículo parte de considerar que el carácter relacional del trabajo docente constituye una dimensión no siempre visibilizada en los estudios sobre el tema o, en ocasiones, reducida al vínculo entre profesores y estudiantes. A lo largo del escrito, se focaliza en las distintas relaciones que establecen los profesores y profesoras en el ejercicio del trabajo docente en la universidad, entendiendo que los otros del trabajo comprenden a distintos sujetos, pero también involucra la relación con la institución universitaria y con particulares conocimientos.

El análisis realizado forma parte de un trabajo de investigación más amplio que tuvo como objeto de estudio el trabajo docente universitario y su evaluación en universidades de Argentina y España1. Se trató de un estudio de casos colectivo de carácter extrínseco o instrumental (Stake, 2005) que permitió abordar la especificidad, la complejidad y la inserción en su contexto de cuestiones relativas al trabajo docente universitario sin la pretensión de establecer generalizaciones. Más que un estudio comparado en sentido estricto, se emprendió un estudio cualitativo con 'sensibilidad comparativa' (Sieder, 2005). Esto es, se buscó reconocer las especificidades de cada caso estudiado (para ello la reconstrucción histórica resulta fundamental) y, a su vez, identificar aquellos actores y dinámicas claves desde el punto de vista trasnacional. Desde esta perspectiva comparativa, Rachel Sieder (2005) propone estudiar aquellos fenómenos que rebasan las fronteras nacionales (por el contexto global y político en el que se insertan), abordando situaciones concretas con una sensibilidad comparativa interdisciplinaria.

En el marco de dicho estudio se realizaron entrevistas semi-estructuradas a sesenta y nueve docentes de dos universidades argentinas Universidad Nacional del Centro de la Provincia de Buenos Aires [UNCPBA] y Universidad Nacional de Misiones [UNaM]) y una española (Universidad de Málaga [UMA]). Se buscó recuperar y explicitar los 'puntos de vista' (Bourdieu, 2007) de profesores de diferentes áreas disciplinares y

1 La investigación fue financiada por la Unión Europea a través de una Beca Full del Programa Erasmus Mundus 'Move on Education', Action 2, EACEA/29/09 Lote 13b. 
distintas categoría y dedicación docente en relación con las peculiaridades del trabajo docente universitario. El criterio de pertenencia disciplinar fue definido a priori a partir del supuesto de la relevancia de la dimensión disciplinaria de la universidad (Becher, 2001). En función de ello, se decidió entrevistar a docentes de cuatro áreas de conocimiento: Ciencias Sociales y Humanidades, Ciencias de la Salud, Ciencias Exactas y Naturales y Arte $^{2}$. Durante el trabajo de campo se incluyeron otros criterios de selección como la categoría y dedicación docente, la antigüedad en la docencia universitaria, el género y la situación de contratación. El análisis de los datos se realizó a través del método de comparación constante propio de la Teoría Fundamentada en el marco de la perspectiva metodológica cualitativa. Con respecto al análisis cabe señalar que las universidades seleccionadas presentan diferencias vinculadas con la historia del sistema universitario en cada uno de sus países, las historias particulares de cada institución, las peculiaridades del contexto político, económico y social, así como con las regulaciones supranacionales, nacionales, regionales e institucionales a las que están sujetas en cada caso. Desde el punto de vista teórico, epistemológico y metodológico, reconocer tales diferencias exigió la adopción de ciertos recaudos interpretativos. Por un lado, no naturalizar aquello que - por la pertenencia a uno de los campos universitarios - resultaba familiar. De este modo, resultó imprescindible ejercer una permanente vigilancia en los escritos para no dar cuestiones por supuestas, para tomar cierta distancia y poder objetivar determinadas situaciones. Por otro lado, requirió indagar en aquellos sentidos, nociones y significados que - al desconocer de otro mundo universitario - no se alcanzaban a comprender. Además, exigió una permanente reconstrucción histórica y contextualización de las políticas, prácticas y actores de modo de no extrapolar lógicas y significados del ámbito conocido hacia aquel que se desconoce y se pretende conocer ${ }^{3}$.

El texto se organiza en seis apartados. Primero, se realizan precisiones conceptuales sobre el trabajo docente en la universidad que permiten identificar su carácter relacional. Segundo, se focaliza en las relaciones entre colegas y las formas que adquiere la cultura del trabajo docente en la universidad. Tercero, se abordan las características que asume la relación del profesorado y los estudiantes. Cuarto, se presentan las cuestiones que emergieron del análisis en lo que refiere a la relación de los docentes con la institución universitaria. Quinto, se atiende a la relación con determinados saberes que se establece en el ejercicio del trabajo docente universitario. Por último, se presentan reflexiones finales a partir de la sistematización del análisis realizado.

\section{El trabajo docente universitario como práctica relacional}

El trabajo docente universitario, concebido como un tipo particular de trabajo sobre los otros (Dubet, 2006), constituye una actividad remunerada que tiene como objetivo explícito transformar a los sujetos. A los docentes universitarios se les paga un salario para socializar a los estudiantes en los códigos de un campo disciplinar específico, 2 A continuación de cada cita textual, se consignarán los siguientes datos de las entrevistas: Institución de pertenencia (UMA, UNCPBA o UNaM), Cargo (Profesor o Auxiliar de docencia), Área disciplinar de pertenencia -Ciencias Sociales y Humanidades (CSH), Ciencias Exactas y Naturales (CExN), Arte (ART) y Ciencias de la Salud (CS), número de entrevista y página de la entrevista en la que se encuentra la cita. Las entrevistas fueron realizadas durante el período febrero 2012-abril 2013.

3 El acompañamiento durante el proceso de investigación de un director español y una co-directora argentina resultó fundamental. Pertenecientes ambos al mundo académico de sus respectivos países, sus observaciones permanentes permitieron ir zanjando las dificultades provenientes de la familiarización con determinados espacios, prácticas y denominaciones y el desconocimiento de otros. En este punto cobra especial sentido el señalamiento que hace Bourdieu acerca del obstáculo que representa para el conocimiento científico tanto el exceso de proximidad como el exceso de distancia. 
en las prácticas de una profesión particular y en las lógicas de la institución universitaria. Este trabajo, tendiente a facilitar la afiliación cognitiva e institucional de los estudiantes en la vida universitaria (Coulon, 1997) y contribuir a su preparación para la vida profesional futura, no se reduce al desempeño de actividades de enseñanza. En el ejercicio del trabajo docente se entraman actividades específicas (consideradas sustantivas del trabajo docente) y actividades de carácter estratégico (tendientes a preservar la cualidad de profesor en el sistema). Actividades todas que se encuentran atravesadas por la tensión entre lo prescrito y lo real del trabajo (Dejours, 2001, 2012). Múltiples actividades involucradas en el ejercicio de la función docente del profesorado, realizadas por los mismos sujetos que también desarrollan tareas de investigación, extensión y gestión. Por ello puede decirse que el profesorado universitario se encuentra en una compleja situación laboral, en un contexto de trabajo que aumenta cada día el nivel de sus demandas. Hace unos años Sancho Gil (2001) se refirió a los 'dos mundos' como una forma de expresar las actividades primordiales del trabajo en la universidad (docencia e investigación). Pasado un tiempo, y dado el creciente lugar que fueron ocupando las actividades de gestión en el tiempo de trabajo de los profesores y profesoras, Sancho Gil, Creus y Padilla Petry (2010) prefieren hablar de "una profesión, tres mundos", haciendo alusión a la triple situación laboral del profesorado (docencia, investigación y gestión). Si se tiene en cuenta, especialmente en las universidades argentinas, la prioridad que se les está otorgando a las actividades de extensión, es que se puede agregar un nuevo mundo a aquella división tripartita.

El trabajo docente constituye una práctica social, históricamente situada que coloca a los profesores en una trama de múltiples dependencias recíprocas. Una red de relaciones con otros, con diferentes saberes, con instituciones particulares y con la sociedad en general. Relaciones atravesadas por configuraciones presentes y pasadas, por condiciones materiales y simbólicas, por representaciones sociales e intereses específicos.

Abordar el carácter relacional del trabajo docente supone reconocer la relevancia de esta dimensión que, por lo general, permanece secreta y poco reconocida (Dubet, 2006). Se señalan a continuación, las distintas'relaciones con otros' que establecen los profesores y profesoras en el ejercicio del trabajo docente en la universidad. Esos otros del trabajo docente comprenden a distintos sujetos -aquí se focalizará en la relación con los estudiantes y con colegas- pero también en la relación con la institución universitaria y con el conocimiento.

\section{Relación con colegas}

Una mirada a la literatura especializada permite afirmar que en los estudios sobre el ejercicio de la práctica docente se priorizan las peculiaridades de un tipo de vínculo particular: la de los docentes con los alumnos. Sin embargo, no puede desconocerse que el ejercicio del trabajo docente supone múltiples relaciones, entre ellas las que se dan en el interior del profesorado: entre profesores diferencialmente posicionados en el espacio universitario. En este apartado se abordarán los aspectos de la relación entre colegas señalados por los profesores de los casos estudiados y que dan cuenta de formas particulares que adopta la cultura de trabajo docente universitario. Se recupera aquí la distinción realizada por Hargreaves (1996) entre 'contenido' y 'forma' de la cultura. 
Para el autor, existen dos dimensiones constitutivas de cualquier cultura: el contenido y la forma. La primera de ellas consiste en las actitudes, valores, creencias, hábitos, supuestos y formas de hacer las cosas fundamentales y compartidas por un determinado grupo. La segunda se refiere a los modelos de relación y formas de asociación características entre los partícipes de la cultura a través de las cuales se realiza, reproduce y redefine su contenido. Del análisis realizado emergen las siguientes formas que adopta la cultura del trabajo docente en la universidad: el individualismo y la balcanización como formas de asociación, la competitividad como clima de trabajo y la creciente virtualización como forma de comunicación.

\subsection{El individualismo y la balcanización como forma de asociación}

Los profesores entrevistados plantean que el ejercicio del trabajo docente universitario requiere de cierta coordinación entre profesores-hacia el interior de las asignaturas, las áreas, las titulaciones, los departamentos, etc.- que permita la concurrencia de esfuerzos en pos de los propósitos institucionales perseguidos. En este sentido, dicen valorar las fórmulas de trabajo colectivo y los intercambios entre los equipos docentes. Sin embargo, reconocen que en las instituciones universitarias no prevalecen las formas de trabajo colaborativo por distintas razones: a) la escasez de tiempo; b) el poco reconocimiento de este tipo de actividades; c) la falta de espacios comunes de trabajo; d) la inestabilidad laboral de los docentes.

La cuestión del tiempo resulta crucial en el análisis de la configuración del trabajo docente. Una profesora novel del área de Ciencias Sociales y Humanidades de la universidad española sostiene: "trabajar coordinadamente es muy difícil (...) [exige] muchísimo tiempo por encima del tiempo que tenemos disponible" (UMA, Profesora novel CSH, Entrevista 5, Pág. 5). Por su parte, un profesor argentino con veinticinco años de antigüedad en el área de Ciencias Exactas y Naturales afirma: "no te reconocen esa labor, parece que eso no es trabajar y eso por lo pronto es injusto" (UNCPBA, Profesor CExN, Entrevista 12, Pág. 4). Las expresiones de los/as docentes permiten advertir la representación del tiempo como 'un bien escaso'. La concepción del tiempo humano apreciado "como un capital que se gasta deprisa y con el que nos vamos también nosotros, (...). Lo cual nos lleva a apelar a no malgastarlo ni dilapidarlo. El tiempo físico fluye y sigue; el nuestro se agota" (Sacristán, 2008, p. 19).

A partir de esa percepción del tiempo como un capital finito, y en función de las situaciones particulares, los docentes deciden emplearlo de distintos modos. Por un lado, trabajar con otros profesores, implica la decisión de invertir tiempo en ello. Por el otro, precisamente por la inversión de tiempo que supone y en relación a los réditos que genera se puede optar por el trabajo individual. Si bien se reconoce la coordinación docente como una práctica relevante y necesaria, se la considera como "tiempo perdido" ya que no es un trabajo reconocido ni valorado. Muchas veces, los logros alcanzados a través del trabajo en equipo no logran compensar los esfuerzos personales (en términos de tiempo y energía dedicada) por lo que el trabajo individual se convierte en una estrategia adaptativa. Esto es, ante las crecientes demandas, las variadas actividades y la presión por los plazos a cumplir que experimentan cotidianamente los profesores y profesoras, el individualismo como forma de trabajo es el resultado de un cálculo estratégico de la inversión eficaz del tiempo y la energía (Hargreaves, 1996). Pero también sucede que 
las formas de trabajo individualista surgen de las limitaciones que imponen las condiciones en las que el mismo se desarrolla. En los tres casos estudiados se mencionó la falta de espacios comunes de trabajo y la inexistencia de una sala de profesores que opere como posibilitador de encuentros. Otro elemento que obstaculiza el trabajo colectivo, y que fue más recurrente en el caso de la universidad española, deviene de la forma de contratación del profesorado. Especialmente, a través de la 'bolsa de interinos'4 por períodos breves (por curso académico o por la duración de la asignatura a impartir). Esto provoca que no se llegue a conocer a los nuevos profesores no sólo por la brevedad e inestabilidad de su experiencia en la universidad sino también porque las condiciones físicas (la falta de espacios comunes) dificultan los encuentros. Se configura así un 'individualismo restringido' que no obedece a un cálculo estratégico, sino que es el resultado de las limitaciones en las condiciones de trabajo. En estos casos se genera una dinámica institucional en la que el colectivo docente constituye una ficción. En este contexto, los proyectos departamentales, los programas de las asignaturas y los propósitos perseguidos por un plan de estudios no trascienden el nivel de lo formal, de lo declarativo. En la práctica, se suele asistir a un conglomerado de prácticas que se superponen, desconocen y hasta llegan a contradecirse. Docentes de las universidades argentinas sostienen que la docencia "...es una profesión muy solitaria dentro de la universidad, vos dictás tus clases y no sabés lo que está pasando en la otra materia" (UNaM, Jefe de Trabajos Prácticos CSH, Entrevista 5, Pág. 3). Además, y en lo que se refiere específicamente a la práctica de enseñanza, suele existir cierta resistencia al trabajo colectivo: "El aula sigue siendo la intimidad docente. El aula sigue siendo un espacio reservado al docente, difícil de compartir, difícil de abrir, difícil de construir con otros" (UNaM, Profesor CSH, Entrevista 4, Pág. 6). Dichas expresiones dan cuenta del carácter solitario que, en ocasiones, adopta el trabajo docente universitario que lleva a ignorar el trabajo de aquellos colegas con los que supuestamente se comparte un propósito institucional.

En función de lo expuesto puede decirse que el individualismo como forma que adopta la cultura del trabajo docente en la universidad puede constituir una respuesta restringida o estratégica a las exigencias y contingencias laborales (la falta de tiempos y espacios, la inestabilidad del cuerpo docente) pero también puede obedecer a la opción de cada profesor de trabajar en solitario. El individualismo puede constituir la manera preferida de estar y ejercer el trabajo docente y en este sentido sería electivo (Hargreaves, 1996).

Ya sea por elección, estrategia o restricción, el individualismo como forma de trabajo docente genera aislamiento, dificulta el encuentro con otros (pares) y limita las posibilidades de construcción colectiva de proyectos institucionales. La cultura balcanizada, expresada en el desarrollo del trabajo en pequeños grupos, se caracteriza por los límites fuertes y duraderos que se establecen entre las distintas partes de la organización, por la identificación personal con los campos que definen esos límites y por las diferencias de poder entre unos campos y otros. Se configura así una 'colegialidad artificial' (Hargreaves, 1996) que, aunque constituye un obstáculo para una colaboración duradera, también puede convertirse en una oportunidad para ésta (Montero, 2011).

\footnotetext{
4 La bolsa de interinos es la expresión que se utiliza para nombrar el listado de titulados acreditados para ejercer la docencia en las universidades españolas. Se trata de una nómina, construida en función de los antecedentes de los candidatos, a la que se recurre para cubrir las plazas vacantes por períodos de tiempo breves.
} 


\subsection{La competitividad como clima de trabajo}

La experiencia de trabajo en la cultura del nuevo capitalismo se ha convertido en una permanente situación de competencia de todos contra todos (Sennett, 2006). En el ámbito de la universidad, no sólo se compite para alcanzar mayor prestigio dentro de una comunidad académica, sino también por la obtención de plazas y de variadas formas de financiamiento (subsidios, becas, etc.). La competitividad (entendida como la capacidad para disputar un capital, la rivalidad por la consecución de un fin) se ha convertido en una eficaz forma de regulación de los sistemas, las instituciones y los sujetos. En un contexto de reducción de la inversión estatal en las universidades públicas y de globalización mercantil de la universidad (Santos, 2005), la competencia aparece como discurso legitimador de las políticas de asignación diferenciada de presupuesto. A nivel estructural, se genera la competencia entre instituciones y en el interior de ellas para la obtención de recursos financieros. A nivel de los sujetos, la competencia entre los docentes se torna propicia para la flexibilidad y la adaptabilidad a las crecientes demandas de trabajo y para la emergencia de estrategias individuales (el 'sálvese quien pueda') que permiten la supervivencia en el ámbito del trabajo. Al respecto, un profesor de Ciencias Sociales y Humanidades de una de las universidades argentinas expresó que el 'clima de trabajo' en su facultad le provoca "dolor de estómago" (UNCPBA, Profesor CSH, Entrevista 5, Pág. 7). Por su parte, un profesor de la universidad española de la misma área disciplinar sostuvo: "A mí me sorprende que hables con profesores de universidad y el 90\% esté harto pero del clima, del ambiente" (UMA, Profesor CSH, Entrevista 3, Pág. 5), mientras que otros colegas de la misma institución sostienen: "Te metes en la universidad y no vez más que trampas, en los departamentos estamos peleándonos por las plazas y yo te voy a hacer la zancadilla aqui" (UMA, Profesor CS, Entrevista 21, Pág. 4), "muchas veces en esta universidad y en todas las envidias son tremendas. $Y$ hay grupos que se hacen daños mutuamente (...) Las relaciones humanas entre el profesorado no son tan buenas como deberían ser" (UMA, Profesor CExN, Entrevista 13, Pág. 5). Las referencias a "trampas", "zancadillas", "peleas por plazas" y "envidias" pueden explicarse por la intensificación de la competencia que lleva a una lucha predatoria y desleal entre colegas. Pero también, es preciso analizar la incidencia de transformaciones de la dimensión temporal en la experiencia del trabajo docente.

Como se planteó en el apartado anterior, la vivencia del tiempo como un bien escaso que no debe malgastarse, atenta contra las formas de trabajo colectivo que requieren una importante inversión de él y que, por lo general, no resulta una actividad suficientemente reconocida. Pero la incidencia de la dimensión temporal no se restringe a las formas de asociación de los docentes, a la decisión de invertir tiempo o no en el trabajo con otros. Las mutaciones de la dimensión temporal en la cultura del nuevo capitalismo también afectan la calidad de los vínculos con los otros. Como sostiene Sennett (2000), el principio del 'nada a largo plazo' que atraviesa en la actualidad a las instituciones, "corroe la confianza, la lealtad y el compromiso mutuos" (p. 22). El cortoplacismo imperante en las nuevas formas de organización del trabajo, con sus formas fugaces de asociación, limita la posibilidad de que madure la confianza informal y se construyan vínculos sólidos y compromisos con los demás. Como sostiene Montero (2011), una cultura de colaboración implica unas relaciones de confianza entre el profesorado, de apoyo mutuo, auto-revisión y aprendizaje profesional compartido. Pero, "para hacer frente a las 
realidades actuales, el desapego y la cooperación superficial son una armadura mejor que el compromiso basado en los valores de lealtad y servicio" (Sennett, 2000, p. 24). Como señala Sennett (2000) la dimensión temporal del nuevo capitalismo es lo que más afecta la vida emocional de las personas; el capitalismo del corto plazo amenaza con corroer aquellos aspectos del carácter que unen a los sujetos entre sí y brindan a cada uno de ellos una sensación de un yo sostenible.

A nivel institucional, las dinámicas individualistas y competitivas ponen en evidencia la dificultad para construir proyectos colectivos. Si se entiende que el trabajo cotidiano de los docentes ha de enmarcarse en un proyecto colectivo ya definido (institucional o a nivel de titulaciones/ departamentos), lo que evidencian la prevalencia del trabajo aislado, el desconocimiento entre colegas y del trabajo que desarrollan y la ausencia de encuentros para articular contenidos u objetivos a nivel horizontal, vertical y transversal; es que tal proyecto no existe. Pero si a la inversa, se considera que ese proyecto colectivo es el resultado del conjunto articulado de las prácticas docentes y tal como plantea Clark (1983) los objetivos institucionales surgen del contacto diario de los pequeños grupos, puede afirmarse que de ese no encuentro entre docentes, de este estado de balcanización en el que trabajan a diario los docentes es poco probable que se puedan construir criterios institucionales compartidos que guíen las acciones. En definitiva, parece tratarse de un círculo vicioso en el que los docentes trabajan individualmente (por razones materiales, estratégicas o electivas y por la debilidad de los vínculos con sus colegas) ya que no perciben "puntos de encuentro" con otros, "sentidos compartidos". Pero, a su vez, la construcción de esos sentidos, de estos intereses colectivos requiere de la posibilidad de encuentros con otros.

\subsection{La virtualización como forma de comunicación}

El análisis de los discursos de los profesores de los tres casos estudiados, permite reconocer la centralidad que cobran las actividades virtuales en la configuración del trabajo docente universitario. La tendencia creciente a la virtualización de las actividades involucra no sólo las referidas a la enseñanza (y que forman parte del trabajo prescrito) y las que constituyen el trabajo estratégico (rellenar formularios, cumplimentar aplicaciones informáticas, realizar informes, etc.), la virtualización del trabajo cotidiano atraviesa la relación con los colegas.

Algunos docentes han señalado que por falta de espacios o por los requerimientos de la tarea (atender el campus virtual) una parte importante de su trabajo lo realizan desde su hogar. El análisis realizado permite advertir que el trabajo docente hoy, más allá de la exigencia del cumplimiento de determinadas horas presenciales, requiere en gran medida del uso permanente del ordenador. La planificación, preparación de trabajos, búsqueda de material, escritura de apuntes, el diseño de un proyecto, la comunicación con los estudiantes, la notificación de calificaciones, el cumplimiento de exigencias burocráticas, etc., requieren el empleo de dicha herramienta. De modo que 'poner el cuerpo' puede resultar necesario sólo en las instancias presenciales: clases, tutorías y acompañamiento a otras instituciones en aquellos espacios que los requieran (prácticas profesionales, prácticas docentes, etc.). El resto de las actividades pueden realizarse desde el propio hogar a través del ordenador en lugar de hacerlo en el despacho de la facultad (si es que se dispone de él). A esto se refirieron varios profesores 
que manifestaron trabajar fundamentalmente en sus casas por no disponer de espacios en la institución, por contar con mejor conectividad a internet que en la universidad (tal como plantearon docentes de la UNCPBA) y porque en el ámbito doméstico cuentan con los recursos necesario para realizar el trabajo (fundamentalmente en áreas como la de Ciencias Sociales y Humanidades). Ante esta situación, resulta interesante aquello que planteara una profesora argentina: "El modelo de universidad va a que cada uno trabaje de forma virtual (...) nos convierte en autómatas, individualistas, con anteojeras cada uno en su casa" (UNCPBA, Profesora CSH, Entrevista 7, Pág. 5).

Lo mencionado permite, por un lado, pensar la forma de trabajo virtual en el marco de un determinado modelo de universidad hegemónico. Si bien la inclusión de las Tecnologías de la Información y la Comunicación (TICs) en el campo educativo puede entenderse como fuente de democratización (de la información, del acceso a los estudios superiores, etc.) no pueden desconocerse las condiciones de desigualdad -entre sistemas nacionales, universidades, facultades y sujetos- en que se las incorpora, así como su vinculación con la 'globalización mercantil de la universidad' (Santos, 2005). Por otro lado, y en el marco de la discusión por las formas de trabajo, las TICs pueden facilitar los intercambios y la conformación de redes académicas (disciplinarias e interdisciplinarias) más allá de los límites de las propias instituciones, pero también al ir sustituyendo paulatinamente los encuentros cara a cara en el interior de las universidades, pueden contribuir a la atomización del profesorado, dificultando formas de organización colectiva.

En la cotidianeidad del trabajo docente, la virtualización como forma de comunicación entre colegas constituye una práctica habitual. Los mensajes dirigidos a un compañero de cátedra, las propuestas de trabajos prácticos que un ayudante comparte con el profesor titular, las calificaciones que el profesor informa al auxiliar para que se las comunique a los alumnos, las sugerencias de algún material de lectura, los informes solicitados por el centro o facultad y que requiere de una elaboración conjunta entre equipos docentes; son discursos y formatos que circulan a través de la red. Es cada vez más frecuente, en el trabajo docente universitario, este tipo de actividades mediadas por la virtualidad por lo que resulta necesario indagar en sus peculiaridades y efectos a nivel de los sujetos particulares y del colectivo docente. ¿Qué es lo que se pierde/cambia/ gana con las actuales formas de 'teletrabajo'? Una pista para pensar esta cuestión puede encontrarse en las características de la comunicación escrita que es la que predomina en las formas de intercambio virtual (correos electrónicos, plataformas virtuales) 5 . La forma de comunicación escrita, en general, requiere más tiempo y mayor detenimiento para la formulación de una idea que la forma de comunicación oral que dispone de otros recursos (ademanes, posturas, etc.). De modo que por una cuestión de economía lingüística se suele restringir a lo imprescindible aquello que se escribe. Dado el mayor esfuerzo que requiere producir un texto escrito, y en el marco de las crecientes demandas de las que son objeto los docentes, escribir o responder un correo electrónico se puede volver una tarea fatigosa (cuantas veces se deja para otro momento y se marca como no leído un e-mail -para tenerlo presente- porque responderlo insume más tiempo del que se dispone en ese momento o porque se decide ya no estar más frente al monitor). Retrasar y esperar el momento oportuno para comunicarse por escrito con un colega puede

5 Más allá que los docentes usen otros recursos como vídeos (que se valen más de la imagen que de la palabra), generalmente la comunicación entre colegas se realiza a través del correo electrónico. 
tener sus ventajas. Esa forma de comunicación exige ser más reflexivo ante aquello que se está escribiendo y por eso se busca la mayor coherencia, se intenta encontrar las palabras más ajustadas a aquello que se quiere transmitir, se aspira a ser lo más claros posible. En fin, se objetiva aquello que se quiere comunicar en el sentido de que se lo hace más consciente, se lo revisa (cuantas veces se relee lo escrito antes de enviar un e-mail). Y como es trabajoso, por lo general, se busca - de manera voluntaria o no- ser concisos, centrarse en aquello que constituye el motivo de la comunicación. En general, los 'asuntos' de los correos electrónicos que circulan entre los docentes universitarios refieren a cuestiones vinculadas con lo académico: planificaciones, bibliografías, contenidos a revisar, entrega de notas, informes para cumplimentar, etc. A lo sumo -si existe la confianza suficient- suele realizarse algún comentario personal, un saludo, pero no mucho más. Detrás de la pantalla, invisibilizados en ese e-mail, quedan los estados de ánimo (el sentir que el trabajo nunca acaba), sensaciones (respecto del sentido del trabajo que se está realizando) anécdotas y situaciones imprevistas (lo que sucedió en una clase, por ejemplo), intuiciones (que se decide no plasmarlas en lo escrito hasta que estén más elaboradas/ pensadas) y sentimientos (el enojo o la alegría por alguna situación vivida en la facultad, en una reunión, etc.; el temor por algo que se está emprendiendo, los nervios por una resolución o notificación que no llega). Se trata de cuestiones vinculadas a la experiencia del trabajo docente que quedan por fuera de un correo electrónico pero que seguramente en una conversación cara a cara, son manifestadas, compartidas ya que esos sentimientos y sensaciones se tornan visibles en la corporeidad. El enojo o la alegría se expresan a través del rostro; el cuerpo da muestras del cansancio, del nerviosismo; los gestos expresan mucho más de lo que se prefiere decir. El cuerpo evidencia aquello que en un correo electrónico se decide no mencionar. $Y$ ese encuentro cara a cara o cuerpo a cuerpo no requiere de un laborioso escrito -que incluya signos de diálogo y comillas- para describir la situación que tanto indignó en una reunión, o para expresar el asombro por una discusión generada entre un grupo de alumnos. El comentario verbal 'al pasar', la opinión sobre 'algo' a un colega, el compartir cara a cara una intuición que no constituye una idea fundada, exime de la forma de expresión más elaborada que exige su explicación por escrito. Precisamente lo que acontece en lo cotidiano del trabajo docente, lo imprevisto, lo que se intuye más que aquello sobre lo que se tiene certeza, lo que se siente, lo que resulta importante y operó como fuente de movilización interior, interpelación pero que no se lo considera tan relevante como para ser escrito en un e-mail; todo eso es lo que queda por fuera de la pantalla, por fuera de la virtualidad. Y no son cuestiones menores ya que se trata de asuntos que atraviesan a los profesores y profesoras como personas, docentes y trabajadores y que al quedar excluidas de las nuevas formas de comunicación quedan invisibilizadas y naturalizadas.

Los enunciados anteriores, lejos de pretender atribuir un carácter maléfico a las tecnologías per se, pretenden pensar la incidencia de la virtualización del trabajo docente en el marco de una trama relacional en la que se conjugan las lógicas del campo, las dinámicas institucionales, las formas de regulación del trabajo docente y las prácticas y percepciones de los sujetos. Es decir, comprender las posibilidades y las dificultades de la virtualización del trabajo docente de manera contextualizada, en relación al conjunto de condiciones, lógicas y prácticas en las que se enmarca. De esta manera, "hay que entender el desarrollo de las nuevas tecnologías fundamentalmente como una 
profundización en la lógica económico-industrial del capitalismo" (Marrero Acosta, 2004, p. 20). En función de esto, en un escenario signado por la descapitalización de la universidad pública donde los docentes son llamados a competir por la obtención de retribuciones económicas, becas, subsidios, etc., donde las nuevas formas de organización del trabajo generan intensificación de tareas, flexibilidad e inestabilidad laboral, donde los programas institucionales se han debilitado (Dubet, 2006), etc. cabe interrogarse en qué medida la virtualización del trabajo docente propicia prácticas y lógicas de trabajo individualistas que atentan contra la organización colectiva del profesorado 6 .

\section{RELACIÓN CON LOS ESTUDIANTES}

Los/as docentes, desde su posición particular en el espacio universitario y en la cotidianeidad de sus interacciones, se relacionan directamente con los estudiantes y van construyendo el rapport pedagógico (Gramsci, 1976) ${ }^{7}$. En el marco de estas interacciones se ponen en juego prácticas y percepciones que van configurando modos singulares de relación. Cada docente y cada estudiante se encuentra con 'el otro de la relación' a partir de un conjunto de esquemas de pensamiento, acción y percepción (Bourdieu, 2002) que le permiten interpretar las situaciones en las que participan al tiempo que las orientan y condicionan.

En el caso de los docentes entrevistados, fueron recurrentes las referencias a los estudiantes en términos de falencias. Salvo algunas excepciones, las representaciones de los docentes respecto de sus estudiantes se construyen a partir de ciertos parámetros sobre cómo deberían ser y se manifiestan en términos de carencias. La falta de conocimientos previos, estrategias de estudio, interés y compromiso político que se atribuye a los estudiantes supone una imagen idealizada-e implícita- de lo que debe ser un estudiante universitario. Tales expresiones encuentran su sustento en representaciones históricas que se han construido sobre estos actores a lo largo del siglo XX y de las que diferentes teorías sociales dan cuenta: el estudiante como representante de una posición privilegiada en la estructura social, portador de autoridad cultural y comprometido políticamente ${ }^{8}$. Si bien tales representaciones persisten como signos de identidad para sectores de la población estudiantil, lo hacen hibridadas por diferentes fenómenos (transnacionalización de las economías, crisis de las ideologías, transformaciones culturales, etc.) que hacen que en la actualidad se asista a una experiencia estudiantil atravesada por la heterogeneidad y la fragmentación (Carli, 2012). De modo que, en las representaciones presentes sobre los estudiantes universitarios se entraman elementos de las experiencias actuales con figuras construidas en determinados momentos históricos. En

6 No se desconoce en este punto que, en contextos de crisis, las tecnologías han sido la plataforma de comunicación de docentes de distintas universidades (muestra de ello son los foros de académicos españoles que se crearon en losúltimos años en el contexto de la crisis política y económica que atraviesa el país). Allí, en la red, profesoras y profesores comparten sus experiencias y sus reivindicaciones. Ameritaría una investigación específica el análisis de las potencialidades de estos dispositivos como espacios de organización colectiva del profesorado.

7 El concepto de rapport pedagógico que aquí se utiliza para dar cuenta de las relaciones entre docentes y estudiantes, no se limita a las relaciones específicamente 'escolares'. Gramsci entiende dicho concepto como aquel vínculo activo, de relaciones recíprocas que se da en toda la sociedad en su totalidad y en cada individuo respecto a los demás.

8 En relación a la construcción histórica de tales representaciones, en el contexto europeo, pueden reconocerse referencias al 'estudiante medio', al 'estudiante normal' o a los 'verdaderos jóvenes' en textos de principios del siglo pasado de filósofos españoles como Ortega y Gasset y Miguel de Unamuno. En Francia, en la tradición sociológica de la década de 1960, surge la figura del estudiante como'heredero', representante de una posición privilegiada en la estructura social y, en tanto producto de la enseñanza universitaria, depositario de variados mecanismos de desigualdad social invisibilizados bajo la ideología del don y del mérito. En América Latina, la producción historiográfica y ensayística, "construyó también un conjunto de representaciones sobre el estudiante universitario que recorren el aristocratismo del Ariel de Rodó, la tradición del reformismo latinoamericano y la politización de los años '70” (Carli, 2006, p. 3). 
los discursos de los profesores entrevistados, las referencias a sus estudiantes en términos de carencias (no les interesa, no saben y no se comprometen) parecen construirse en función de determinadas imágenes de lo que es un estudiante universitario: 'joven innovador', 'heredero' y 'militante'.

Los nuevos estudiantes que habitan la universidad, repartiendo su tiempo entre el trabajo y el estudio, que no necesariamente son jóvenes (en el sentido tradicional de corresponder a determinada franja etaria), que establecen relaciones con el conocimiento y prácticas de lectura diferentes a las de sus profesores y cuyos intereses intelectuales y políticos no se circunscriben al ámbito universitario, ponen en jaque las representaciones sobre el estudiante ideal y el estudiante real. Se trata, en términos de Corea y Lewkowicz (2004), del desacople entre el alumno supuesto y el alumno real que efectivamente habita la universidad. Y, por otra parte, implica una tensión entre el horizonte de expectativas de los docentes respecto de la formación universitaria y las representaciones que construyen sobre los estudiantes en sus experiencias de trabajo en el terreno de la enseñanza (Carli, 2012).

En el interjuego entre lo que se espera de los estudiantes y de cómo son percibidos, los docentes se encuentran ejerciendo actividades que no forman parte de su trabajo prescrito pero que resultan sustantivas para el aprendizaje de los estudiantes. Por ejemplo, la enseñanza de conocimientos que se requieren para el abordaje de los contenidos específicos de la asignatura impartida. Al considerar que los estudiantes no poseen estos saberes previos, los docentes entrevistados manifestaron tener que dedicar tiempo a este tipo de contenidos ya que resultan necesarios para el aprendizaje de lo que curricularmente deben aprender en las asignaturas que dictan. Lo mismo se señaló en relación con las estrategias de estudio; los docentes manifiestan dedicar tiempo y energías a la orientación de los estudiantes en lo referido a búsquedas bibliográficas, lectura e interpretación de textos y el uso de diferentes técnicas que les permitan identificar información relevante. Asimismo, sostienen que el desarrollo de debates y situaciones de argumentación demanda mucho acompañamiento de su parte. En este sentido, lo disciplinar no siempre ocupa el centro de atención en las prácticas de enseñanzas. Y desde un punto de vista más general, sin restringir las interacciones de los docentes con los estudiantes a los espacios y tiempos del aula y a los contenidos prescritos curricularmente, los profesores y profesoras acompañan y orientan a sus alumnos en aspectos organizativos y administrativos de la institución universitaria. Brindar información acerca de los procedimientos para realizar determinado trámite, indicar a quiénes deben dirigir ciertas consultas, incluso escuchar y aconsejar a los alumnos en cuestiones que exceden lo académico son algunas de las actividades mencionadas. De modo que las relaciones entre docentes y estudiantes no están mediadas únicamente por el contenido, por la cuestión disciplinar. El reconocimiento de las identidades más allá de los roles institucionales desempeñados -el de docentes o el de estudiantes-y de los sujetos como sujetos sociales, adultos y jóvenes históricamente situados y sujetos de deseo, permite advertir que lo que fluye, circula en las relaciones pedagógicas no se circunscribe a lo académico. Deseos, sentimientos, sensaciones y expectativas las atraviesan y configuran.

El tránsito de los estudiantes por la universidad supone no sólo el encuentro -o desencuentro- con un campo disciplinar específico sino también con una cultura institucional que requieren del aprendizaje de sus lenguajes, sus saberes y sus costumbres. 
Durante su experiencia estudiantil, que más que la expresión lineal de una racionalidad consciente, constituye el producto recursivo de dudas, temores, equivocaciones y pasiones (Malinowski, 2008), los estudiantes emprenden un doble aprendizaje. Por un lado, un aprendizaje específico, propio de la cultura disciplinar a la que se está ingresando y por otro un aprendizaje general que involucra las características de la universidad como institución y que implica construir el oficio de estudiante universitario. De esta manera, en este 'aprender a ser estudiante' parece que cada alumno va transitando una serie de momentos que - con tiempos diferentes y sin significar una suerte de evolución naturalle permiten paulatinamente ir apropiándose de las reglas de juego de la propia universidad $^{9}$. Afiliarse consiste en apropiarse de las características multidimensionales -tanto administrativas como cognitivas- de la institución universitaria y en edificar un conjunto de referentes en el seno del mundo universitario. Se trata, en términos de Bourdieu (2007), de la construcción del sentido del juego. En este arduo proceso de construcción del oficio de estudiante, la fase de afiliación juega un papel importante ya que a medida que los estudiantes van conociendo las reglas de juego del mundo universitario, ellos mismos se van reconociendo como parte de ese nuevo universo, incorporando sus prácticas y forjándose un 'habitus de estudiante' que se constituirá en principio orientador de sus acciones. En este sentido, el habitus, entendido como una disposición a actuar, percibir, pensar y sentir de determinada manera (Bourdieu, 2008), se irá construyendo paulatinamente en las interacciones que el alumno establezca en el marco de la institución, en general; y de un campo de conocimiento específico, en particular.

Una cuestión relevante que merece ser tenida en cuenta en el análisis de las relaciones de los docentes con los estudiantes tiene que ver con la simultaneidad del proceso de afiliación de los estudiantes y el que emprenden los profesores noveles en su acceso a una posición particular como docente. Es decir, si ingresar a la universidad significa comenzar a transitar un ambiente cultural específico, resulta necesario un proceso de socialización en el que los futuros miembros de una comunidad académica (sean estudiantes o docentes) vayan construyendo progresivamente su sentimiento de pertenencia, de identidad y compromiso personal con la misma. Si se recuperan las categorías de Coulon (1997) y se las emplea para analizar la situación de los docentes universitarios noveles puede decirse que tales actores se encuentran en un momento de su carrera académica en el que, entre otras cosas, necesitan aprender las reglas de juego de ese nuevo mundo al que se están incorporando. Porque a pesar haber transitado por varios años la universidad en carácter de estudiantes, la nueva posición ocupada como docentes los ubica en un lugar diferenciado respecto del conocimiento (que ahora debe ser enseñado), de los estudiantes (que antes eran pares), de los docentes (que ahora son colegas) y de la institución (con la que existe un vínculo contractual y funciones que cumplir). De modo que cuando los docentes noveles acompañan a los estudiantes -fundamentalmente de los primeros años - en la etapa de extrañamiento y los orientan en su inserción y aprendizaje de ese mundo cultural específico que es la universidad para que alcancen la afiliación, ellos mismos están atravesando un proceso de aprendizaje de las nuevas reglas del juego, ellos mismos están intentando afiliarse como docentes a la

9 Coulon (1997) señala tres etapas que prosiguen a la entrada a la universidad: 1) un tiempo de extrañamiento, que supone la inserción a un ámbito desconocido y la ruptura con las normas y costumbres anteriores, propias del mundo que se acaba de abandonar; 2) un tiempo de aprendizaje, que implica un proceso de adaptación a las nuevas pautas institucionales y el descubrimiento de las ambigüedades entre las antiguas y las nuevas reglas; y 3) un tiempo de afiliación, en el que el estudiante logra dominar las nuevas reglas de juego e interpretar los significados institucionales. 
institución universitaria.

Por último, cabe mencionar respecto de la configuración de la relación entre los estudiantes y los profesores universitarios, que en losúltimos años el trabajo docente universitario ha ido asumiendo la forma de un trabajo "a demanda". Más allá del ideal pansófico comeniano, los/ as docentes responden diferencialmente a las crecientes consultas individualizadas que los estudiantes realizan en los espacios y tiempos de tutorías o a través del correo electrónico. Esto último, constituye una de las expresiones de la mediatización del trabajo docente por el uso de las TICs. Sostienen los docentes: "yo me encuentro que un sábado se me ocurre abrir el correo electrónico, me puedo encontrar diez correos de alumnos que están estudiando en sus casas (...) pues "profesor tengo tal duda"” (UMA, Profesor CExN, Entrevista 12, Pág. 2); "muchas veces que en casa ya es tarde y te pones a mirar cosas y resulta que hay alumnos que están conectados, que es el momento que están estudiando. Y bueno, si en ese momento puedo resolver una duda" (UMA, Profesor CExN, Entrevista 14, Pág. 6). No puede desconocerse en este punto la centralidad otorgada a los estudiantes en los procesos de enseñanza y aprendizaje que, en el marco de la lógica mercantil que atraviesa al campo universitario, parece responder a la concepción del estudiante como cliente. En el marco del nuevo espíritu del capitalismo en el que el 'cliente es el rey' (Boltanski y Chiapello, 2002), para algunos docentes la virtualización de la enseñanza abre canales de comunicación que permiten un mayor acompañamiento a los estudiantes. Para otros, dicha tendencia configura un modo de relación en el que falta lo esencial, 'el factor humano'. Como se advierte, al igual que en las relaciones entre colegas, las TICs ocupan un papel importante en la configuración de la dimensión relacional del trabajo docente.

\section{Relación con la institución}

Ser docente universitario supone establecer ciertas relaciones con la institución universitaria y la organización de pertenencia. Una de ellas, es la que deviene de las particulares formas de contratación. Cabe señalar que en el mundo del trabajo general y en el campo universitario en particular, han aflorado formas de contratación por períodos determinados, a tiempo parcial y sin garantía de continuidad. De los casos estudiados estas situaciones se tornan más patentes en la universidad española. Las actuales formas de contratación llevan a los docentes universitarios, fundamentalmente a los que recién comienzan, a experimentar una permanente situación de inestabilidad laboral que repercute, no sólo en su vida personal y en el tipo de vínculo que se establece con los estudiantes y los colegas, sino en la relación que se establece con la institución. En Argentina, la periodicidad de los concursos atenta contra la permanencia en un cargo y las posibilidades de promoción en la carrera docente se ven amenazadas por la falta de financiamiento para la sustanciación de los concursos docentes ${ }^{10}$. Una profesora plantea: "Al final el propio sistema termina desmotivándote porque uno dice: yo me voy a comer la cabeza, voy a estar dándole vueltas, perdiendo mi tiempo, no dedicándole a mi familia, mis hijos, para qué" (UMA, Profesora novel CExN, Entrevista 16, Pág. 6). En un contexto en el que se suele exigir a los docentes mayor compromiso con la institución,

10 El recientemente aprobado Convenio Colectivo de Trabajo (CCT) genera modificaciones en las condiciones de trabajo de los docentes universitarios, fundamentalmente en aquellas vinculadas a la estabilidad laboral. El convenio plantea que cada universidad diseñará su mecanismo institucional de evaluación periódica individual del cual estará sujeta la permanencia en el cargo que cada docente ordinario o regular hubiere alcanzado. 
cabe preguntarse "¿Cómo habría alguien de comprometerse con una institución que no se compromete con uno?" (Sennett, 2006, p. 167). Y es que, en el actual escenario de trabajo, signado por la inestabilidad y la flexibilidad laboral, el no comprometerse puede entenderse como una forma de sentido del juego (Bourdieu, 2007); como la respuesta estratégica que permite cumplir, al menos mínimamente, con el compromiso intermitente exigido por la institución intentando permanecer en ella el mayor tiempo posible. Y es que no resulta redituable (en términos de inversión de tiempos y energías) ni beneficioso para la salud mental comprometerse y construir vínculos de confianza en un espacio incierto que deja a los sujetos a la deriva (Sennett, 2000, 2006). Lo novedoso de esta situación, "lo que hoy tiene de particular la incertidumbre es que existe sin la amenaza de un desastre histórico; y en cambio está integrada en las prácticas cotidianas de un capitalismo vigoroso" (Sennett, 2000, p. 30). La experiencia de la inestabilidad laboral que debilita los vínculos con otros y el compromiso con la universidad, dificulta -sobre todo para los que recién comienzan-su afiliación institucional (Coulon, 1997).

Otro tipo de relación que se establece con la organización universitaria tiene que ver con las disposiciones de los mecanismos de regulación. En el caso de la universidad española, que lleva más de una década de experiencia en la implementación de las directrices emanadas del denominado Proceso de Bolonia, la mayoría de los profesores y profesoras vienen transitando un conjunto de cambios que afectan su trabajo docente cotidiano: incremento de las evaluaciones formales de su labor, mayores exigencias en cuanto al cumplimiento con formatos específicos de planificación de la enseñanza, requerimientos de desarrollo de determinadas metodologías y prácticas consideradas innovadoras, etc. Realizados estos esfuerzos y, pasado un tiempo, viendo incumplidos - total o parcialmente - muchos de los propósitos que fueron baluarte del Espacio Europeo de Educación Superior (disminución de la relación profesor-alumnos para posibilitar una enseñanza centrada en el estudiante, acompañamiento personalizado, evaluaciones procesuales, etc.) los docentes manifiestan que aquellas intenciones "quedaron en papel mojado". Ante la creciente demanda de actividades (especialmente de tipo burocráticas) que restan tiempo y energías para aquello que se considera sustantivo del trabajo docente, lo que surge es la superficialidad del trabajo como estrategia que permite cumplir con las obligaciones sin mayores compromisos. En este sentido, las prescripciones y la rendición de cuentas otorgan una particular configuración a la relación del profesorado y la organización universitaria en el ejercicio de la función docente.

Por último, en cuanto a las formas que adquiere la relación de los docentes con la institución, cabe señalar la relevancia de un proyecto institucional que permita a los profesores dotar de sentido y orientación su trabajo cotidiano. Los/as docentes de las tres universidades estudiadas señalaron la necesidad de contar con proyectos institucionales que integren los esfuerzos y los orienten en pos de la consecución de determinados objetivos. A ambos lados del Atlántico, los docentes dicen percibir la falta de propuestas político-académico-pedagógicas que orienten "hacia dónde caminar", que "integren $y$ articulen esfuerzos y permitan planificar a largo plazo" y, de esta manera, responder a los requerimientos de la sociedad. En este contexto, fueron varios los que manifestaron sentirse desconcertados, sin saber muy bien lo que se espera de ellos. En esas expresiones emerge la imagen de la 'deriva' señalada por Sennett (2000), de la incertidumbre, del debilitamiento de los proyectos institucionales como obstáculo para otorgar sentido 
al trabajo docente. Esta falta de sentido, la opción por no comprometerse, incluso la sensación de agotamiento ante un trabajo ve amenazadas sus fuentes de satisfacción pueden comprenderse en el marco del debilitamiento del programa institucional de la universidad. Señala Dubet (2006) que todo lo que el programa institucional estaba en condiciones de ligar y confundir se separa paulatinamente, haciendo aparecer las lógicas de acción cada vez más autónomas. Los actores ya no entran en las instituciones armados de sólidas motivaciones tradicionales; el desencantamiento del mundo priva a las instituciones de los consuelos que hacían soportable la experiencia de los actores, la autoridad dejó de ser natural y sagrada para requerir de un trabajo de justificación permanente. De esta manera, las sensaciones de desorientación de los profesores y la dificultad que dicen encontrar para otorgarle sentido a su trabajo pueden entenderse en el marco del debilitamiento del programa institucional que generó la descomposición de elementos y representaciones que antes se percibían como integrados. El debilitamiento del programa institucional hace que los valores orientadores de las prácticas pierdan unidad, que la vocación choque contra los requerimientos de eficacia profesional, contra los constreñimientos de organizaciones más lábiles y complejas, y que la creencia en una continuidad entre socialización y subjetividad ya no resulte evidente (Dubet, 2006). En este sentido resulta significativo pensar los actuales cambios en las experiencias y relaciones del trabajo docente universitario en el marco de las mutaciones internas de los programas institucionales y de las modalidades de socialización.

Enders y Kaulisch (2006) señalan el proceso de transformación al que han asistido las universidades desde la década de 1980 y que las han acercado como organización a un modelo empresarial. En ese contexto, argumentan que las tendencias hacia la mercantilización, el gerencialismo y la internacionalización generan una combinación de rasgos viejos y nuevos, a partir de la cual las carreras académicas 'se entrelazan con' y a la vez 'se separan de' la institución. Por un lado, el crecimiento de la actividad intersectorial, la movilidad internacional de los profesores, el aumento del personal auxiliar y de tiempo parcial y la erosión de conceptos tradicionales como el de tenure (profesorado permanente) constituyen condiciones que desvinculan a los profesores del ámbito organizacional de trabajo. Por el otro, la introducción de nuevas formas de gestión de las universidades tiende a alinear las actividades del profesorado en función de las necesidades e intereses organizacionales y crea mercados internos de trabajo vinculando a los profesores con la institución. De ahí que se dé un doble movimiento entre tendencias que contribuyen a configurar carreras nómadas, sin fronteras y agenciadas por los propios sujetos y políticas y prácticas universitarias que instituyen regulaciones tendientes a vincular a los académicos a la institución.

\section{Relación con el conocimiento}

Como ya se ha venido planteando, el mundo universitario no constituye un espacio homogéneo. En la construcción de la heterogeneidad que lo caracteriza, la dimensión disciplinaria ocupa un papel relevante. Clark afirma que "la materia del conocimiento -más aún, la de tipo avanzado- es la médula de los propósitos y la esencia de cualquier sistema de educación superior" (Clark, 1983, p. 35). El conocimiento es la materia prima en función de la cual se estructuran las dinámicas institucionales y las prácticas académicas. $Y$ es precisamente el personal académico el que lo manipula de diversos modos 
para descubrirlo, conservarlo, depurarlo, transmitirlo o aplicarlo. En este sentido, el autor afirma que lo que hace un profesor universitario es circular con un paquete de conocimiento, general o específico, en busca de la manera de aumentarlo o enseñarlo a los demás. De esta sustancia crecientemente especializada y autónoma se derivan ciertos aspectos de forma, agrupándose las tareas y los trabajadores de acuerdo con diversos paquetes de conocimiento.

Becher (2001, p. 13) se interesó en "trazar un mapa del multicolor territorio del conocimiento académico y por explorar las diversas características de quienes lo habitan y lo cultivan". El autor afirma que en el campo universitario coexisten tribus ligadas a determinados territorios (territorios cognitivos) que operan con una determinada tradición cognitiva (categorías de pensamiento) y códigos de comportamiento (Clark, 1983). Cada una de esas tribus posee sus propias tradiciones, costumbres y prácticas; conocimientos, creencias y principios morales; formas lingüísticas y simbólicas de comunicación y significados que unifican a quienes forman parte de ella y que deben ser adquiridas por quienes pretenden pertenecer a la tribu (Araujo, 2008). Ingresar a la universidad en carácter de estudiante o de docente supone socializarse en una cultura institucional peculiar y en una determinada cultura académica. Aunque se hayan transitado varios años como alumno en una determinada carrera universitaria que brinda las herramientas necesarias para la apropiación de los saberes de la cultura disciplinar de pertenencia, el pasaje a la categoría de docente implica nuevos aprendizajes en relación a la lógica de la disciplina. Ser profesor de determinada área disciplinar no constituye una extensión de las prácticas ejercidas y de las identidades construidas durante la experiencia estudiantil. Los procesos de aprendizaje y afiliación, tanto cognitiva como institucional (Coulon, 1997), que sirvieron para la incorporación de los estudiantes a ese nuevo mundo que es la universidad necesitan ser puestos en marcha nuevamente para habitar el mundo universitario desde otra posición: la de profesor o profesora. Ser docente universitario implica nuevas y diferentes relaciones con la institución, con los estudiantes, con aquellos que de maestros pasan a ser colegas y con un conocimiento que ahora debe ser enseñado.

Las prácticas de enseñanza adquieren particulares configuraciones según los campos disciplinares y las herramientas metodológicas de que se dispone para llevarlas adelante. Además de los conocimientos disciplinares, requiere ciertos saberes didácticos y pedagógicos (en lo relativo a la organización de las actividades en clases, el uso de recursos didácticos, la motivación de los estudiantes, el fomento de la participación, etc.) que no todos los docentes universitarios han construido. Al respecto, un profesor del área de Ciencias Exactas y Naturales plantea: "un docente tiene que saber explicar, tiene que saber algo de pedagogía o saber manejar una clase, pero ¿Cómo le exigís eso a una persona que no tiene formación en ese sentido?" (UNCPBA, Profesor novel CExN, Entrevista 15, Pág. 2). Un colega del área artística afirma que "hay muchas carreras que no le da la formación necesaria al docente para poder llevar adelante bien sus clases" (UNCPBA, Profesor ART, Entrevista 22, Pág. 2). Por su parte, una profesora de Ciencias Sociales y Humanidades critica la ausencia de formación pedagógica en su formación de grado y considera un absurdo que no se exija en el nivel universitario la capacitación docente que sí se requiere en otros niveles (UNCPBA, Ayudante de docencia CSH, Entrevista 3, Pág. 3). Los docentes ponen en evidencia que el sistema los habilita para desempeñar una función - la función docente- para lo cual no todos fueron preparados. 
De modo que puede afirmarse que, en relación a la formación pedagógica, los/as docentes universitarios se encuentran en desigualdad de condiciones. En muchas áreas, los profesores cuentan fundamentalmente con la solvencia en el conocimiento de su disciplina. Son más bien intuiciones las que tienen acerca de las características de sus estudiantes y las peculiaridades de los procesos de aprendizaje, así como de las limitaciones y potencialidades de determinadas estrategias de enseñanza. En otras áreas disciplinares, sin embargo, se considera indispensable poseer esos conocimientos para poder llevar adelante la práctica de enseñanza. Éstas son fundamentalmente áreas en las que el sujeto de aprendizaje o la práctica de enseñanza forman parte de su objeto de estudio. De esta manera, ocurre que profesores universitarios en el ejercicio de su función docente deben llevar adelante una tarea cuya formación no se encuentra previamente garantizada ya que en su mayoría fueron preparados para desempeñarse como biólogos, ingenieros, médicos, contadores, etc. Estas tensiones en las relaciones con el conocimiento (disciplinar y pedagógico) que se establece en el ejercicio del trabajo docente, remite a la cuestión de la identidad de los profesores y profesoras universitarios (Zabalza, 2009; Lucarelli, 2004) y a las marcas de la profesión de origen que hacen de la docencia una 'categoría residual' (Chiroleu, 2002). Y es que la profesión académica nuclea a graduados que, una vez obtenidas sus titulaciones, no ejercen la profesión para la que fueron formados más allá del espacio universitario, sino que desarrollan su trayectoria profesional y laboral en cargos docentes en las propias universidades (Edelstein, 2012). Otra cuestión a señalar respecto de la relación con el conocimiento que supone el ejercicio del trabajo docente, emerge de la vinculación de la necesidad de formación permanente y la lógica de mercado que impera en el capo universitario. Sostiene un profesor: "Vos hiciste un grado, pero después hiciste un máster, después hiciste un doctorado, después vendrá un pos doc y estás corriendo siempre" (UNCPBA, Profesora CSH, Entrevista 7, Pág. 7). Los docentes se refirieron a la tendencia a "correr detrás de los títulos", "ir detrás de la zanahoria". Así, si bien la titulación académica constituye un requisito para el ingreso a un cargo docente, en los últimos años se ha configurado un mercado universitario de posgrados, congresos, cursos, seminarios, etc. que, al igual que plantea Collins (1989) en su análisis de la sociedad credencialista, no garantizan per se conocimientos y habilidades. Como manifestaron los profesores y profesoras entrevistados, muchas veces la decisión de realizar un posgrado, asistir a un congreso o cursar un seminario obedece más a una estrategia por la obtención de esa moneda de cambio que es la credencial, que a una necesidad o interés formativos. Por último, cabe señalar que la pertenencia a un campo disciplinar, entendiendo la disciplina como una construcción socio-histórica- ubica diferencialmente a sus integrantes en el espacio universitario, generando condiciones de desigualdad en términos de reconocimiento y prestigio. Socialmente, no es lo mismo ejercer el trabajo docente siendo médico que pedagogo. No goza del mismo prestigio un profesor del área de Ciencias Exactas y Naturales o Medicina que otro de Ciencias Sociales, Humanidades o Arte. En este sentido, la relación con el conocimiento en el ejercicio del trabajo docente está atravesada por dinámicas de legitimación de la estratificación ocupacional y la reproducción social. Los mecanismos de cierre social de determinadas comunidades profesionales y su auto-legitimación mediante la ideología técnico-funcionalista que argumenta la necesidad de una larga y selectiva formación especializada se erigen como estrategias de protección del gremio (Collins, 1989) que generan representaciones sociales sobre tales comunidades. 


\section{REFLEXIONES FINALES}

La trama de múltiples relaciones en que los profesores universitarios desarrollan su quehacer diario, impide hablar del trabajo docente como una categoría homogénea. Su multidimensionalidad y especificidad, las condiciones diversas en las que se desarrolla, así como su carácter relacional, hacen del trabajo docente universitario un dominio diverso (Scott, 2008), una práctica compleja (Morín, 1994) de límites difusos (Sancho Gil, 2001). Las relaciones que en el ejercicio de trabajo docente se establece con la institución, colegas, estudiantes, conocimientos y saberes están permeadas por el tipo de relación que la universidad mantiene con la sociedad.

El análisis realizado permite advertir que el carácter relacional del trabajo da lugar a la configuración de una serie de tensiones. En primer lugar, puede decirse que el trabajo docente es considerado por los sujetos como una práctica social de carácter colectivo, que requiere del trabajo conjunto y de criterios compartidos. Sin embargo, se reconoce que las condiciones y regulaciones que configuran el trabajo cotidiano, propician prácticas y dinámicas que atentan contra la conformación de vínculos de confianza, solidaridad y compromiso con otros. Es en este sentido, que se puede afirmar que el trabajo docente en la universidad está atravesado por la tensión entre lo individual y lo colectivo.

En segundo lugar, se reconocen tendencias que tienden a centralizar a los docentes en torno a la universidad como organización y fuerzas que fragmentan las identidades en torno a campos disciplinares específicos. El análisis desde la perspectiva organizacional y la recuperación de la dimensión disciplinaria permite comprender el trabajo docente en el marco del interjuego entre lo general y lo particular propio de ese universo simbólico organizacional que reconoce a todos los integrantes como miembros de la universidad, y aquel universo simbólico disciplinar que fragmenta la construcción de identidades alrededor de matrices disciplinarias y campos profesionales con prácticas idiosincrásicas (Araujo, 2008). En este punto, el trabajo docente en la universidad está atravesado por la tensión entre la pertenencia disciplinar y la pertenencia a una organización.

En tercer lugar, se reconoce una doble tendencia en relación a las carreras académicas de los docentes. Por un lado, se ancla a los docentes a las universidades a través de formas de gestión de las universidades que buscan orientar las actividades de los profesores en función de sus intereses. Por el otro, la internacionalización, la movilidad y el aumento del tiempo parcial como forma de contratación los desvin culan de las instituciones fomentando las carreras nómadas. En este sentido, el trabajo docente está atravesado por la tensión entre fuerzas que lo anclan a una organización y fuerzas que los desvinculan de ellas propiciando carreras sin fronteras. De modo que a la tensión -desde un punto de vista sincrónico- entre las fuerzas disciplinares que tienden a la fragmentación del trabajo docente universitario y las fuerzas organizacionales que tienden a su integración, se le puede agregar — desde un punto de vista diacrónico- la tensión entre fuerzas que desvinculan las trayectorias de los docentes de la propia institución y las que las pretenden ligarlas orientándolas en función de sus finalidades e intereses.

Por último, cabe señalar que el análisis del carácter relacional de la docencia universitaria desde la categoría de trabajo permitió abordar múltiples dimensiones que configuran dicha práctica y reconocer tensiones que la atraviesan. Cuestiones éstas que 
hacen a la complejidad, heterogeneidad y especificidad del trabajo docente en la universidad y que no siempre son reconocidas. Y esto porque "el trabajo como tal ha sido y aún es, escaso tema de análisis y reflexión en la vida universitaria" (Martínez, 2013, p. 48). En este sentido, el abordaje que aquí se propuso del trabajo docente como práctica relacional buscó visibilizar el entramado de vínculos que lo van configurando y que coloca a los profesores y profesoras universitarios en una red de múltiples relaciones con otros sujetos, saberes e instituciones.

\section{REFERÊNCIAS}

Araujo, S. Formación universitaria y éxito académico: disciplinas, estudiantes y profesores. Tandil: Universidad Nacional del Centro de la Provincia de Buenos Aires, 2008.

Becher, T. Tribus y territorios académicos. La indagación intelectual y las culturas de las disciplinas. Barcelona: Gedisa, 2001.

Boltanski, L. y Chiapello, Ė. El nuevo espíritu del capitalismo. Madrid: Ediciones Akal, 2002.

Bourdieu, P. El oficio del sociólogo. Buenos Aires: Siglo XXI Editores, 2002.

Bourdieu, P. El sentido práctico. Buenos Aires: Siglo XXI Editores, 2007.

Bourdieu, P. La práctica de la sociología reflexiva (Seminario de París). En Bourdieu, P. y Wacquant, L. Una invitación a la sociología reflexiva. Buenos Aires: Siglo XXI Editores, 2008, pp. 267-317.

Carli, S. La experiencia universitaria y las narrativas estudiantiles. Una investigación sobre el tiempo presente. Revista Sociedad, Nº 25, pp. 29-46, 2006.

Carli, S. El estudiante universitario. Hacia una historia del presente de la educación pública. Buenos Aires: Siglo XX Editores, 2012.

Chiroleu, A. La profesión académica en Argentina. Boletín PROEALC, Síntesis Especial América latina, mayo. 2002. Extraído el 20 de noviembre, 2015 de http://www. proealc.uerj.br.

Clark, B. El sistema de educación superior: Una visión comparativa de la organización académica. México: Nueva Imagen/Universidad Futura/UAM, 1983.

Collins, R. La sociedad credencialista. Sociología histórica de la educación y la estratificación. Madrid: Ediciones Akal, 1989.

Corea, C. y Lewkowicz, I. Pedagogía del Aburrido: Escuelas destituidas, familias perplejas. Buenos Aires: Editorial Paidós, 2004.

Coulon, A. Le métier d'étudiant: I' entrée dans la vie universitaire. Paris: Presses Universitaires de France, 1997.

Dejours, C. Subjectivité, travail et action. La Pensée N³28, 2001, pp. 7-19.

Dejours, C. Del trabajo a la subjetividad. Trabajo Vivo. Tomo I. Sexualidad y Trabajo. Buenos Aires: Editorial Topía, 2012.

Dubet, F. El declive de la institución. Profesiones, sujetos e individuos en la modernidad. Barcelona: Editorial Gedisa, 2006. 
Edelstein, G. Un debate vigente y necesario: la formación docente en las universidades. Lorenzatti, M. (Coord.) Construcción cooperativa de políticas y estrategias de formación de docentes universitarios en la región. Córdoba: Universidad Nacional de Córdoba, 2012, pp. 9-16.

Enders, J. \& Kaulisch, M. The Binding and Unbinding of Academic Careers. In Teichler, U. (Ed.) The Formative Years of Scholars. London: Portland Press (Wenner-Gren International Series, Vol. 83), 2006, pp. 85-96. Extraído el 20 de noviembre, 2015 de http:// www.portlandpress.com

Gimeno Sacristán, J. El valor del tiempo en educación. Madrid: Ediciones Morata, 2008.

Gramsci, A. La alternativa pedagógica. Barcelona: Editorial Nova Terra, 1976.

Hargreaves, A. Profesorado, cultura y posmodernidad. Cambian los tiempos, cambia el profesorado. Madrid: Ediciones Morata, 1996.

Lucarelli, E. Prácticas innovadoras en la formación del docente universitario. Revista Educaçao, año XXVII, Núm. 3, pp. 503-524, 2004.

Malinowski, N. Diferenciación de los tiempos estudiantiles e impacto sobre el proceso de afiliación en México. Revista latinoamericana de ciencias sociales, Niñez y Juventud, Vol. 6, N², pp. 801-819, 2008.

Marrero Acosta, J. Sociedad de la información y dinámica mediadora de la universidad. Revista Qurriculum No 17, pp. 17-46, 2004.

Martínez, D. El trabajo en la universidad. Pequeña introducción a un texto para dar batalla. Revista Espacios en Blanco N²3, pp. 45-72, 2013.

Montero, L. El trabajo colaborativo del profesorado como oportunidad formativa. CEE Participación Educativa N 16, pp. 69-88, 2011.

Morin, E. Epistemología de la complejidad. Fried Schnitman, D. (Comp.) Nuevos paradigmas, cultura y subjetividad. Buenos Aires: Editorial Paidós, pp. 421-442, 1994.

Sancho Gil, J. Docencia e investigación en la universidad: una profesión, dos mundos. Educar, N²8, pp. 41-60, 2001.

Sancho Gil, J., Creus, A. y Padilla Petry, P. Docencia, investigación y gestión en la Universidad: una profesión, tres mundos. Praxis Educativa, Vol. XVI, No 14, pp. 17-34, 2010.

Santos, Boaventura de Sousa (2005). La universidad en el siglo XXI. Para una reforma democrática y emancipadora de la universidad. Buenos Aires: Miño y Dávila, 2005.

Scott, P. ¿Divergencia o convergencia? Las relaciones entre docencia $\mathrm{e}$ investigación en la educación superior de masas. Barnett, R. (Ed.) Para una transformación de la universidad. Nuevas relaciones entre investigación, saber y docencia. Barcelona: Editorial Octaedro, pp. 75-91, 2008.

Sennett, R. La corrosión del carácter. Las consecuencias personales del trabajo en el nuevo capitalismo. Barcelona: Editorial Anagrama, 2000.

Sennett, R. La cultura del nuevo capitalismo. Barcelona: Editorial Anagrama, 2006. 
Sieder, R. Entrevista a Rachel Sieder. La tendencia hacia más estudios comparados es lógica si pensamos en el momento global y político en el que estamos. Revista Sistemas Judiciales № 8- Posibilidades y límites de la comparación. pp. 61-64, 2005.

Stake, R. Investigación con estudios de caso. Madrid: Ediciones Morata, 2005.

Zabalza, M. Á. Ser profesor universitario hoy. La cuestión Universitaria № 5, pp. 69-81, 2009.

Data de atorização de publicação: 28/11/2018

DOI: http://dx.doi.org/10.5965/25944630312019009 